\title{
PERDA DE CORTE DA LIMA PROTAPER QUANTO A ESTERILIZAÇÃO E NÚMERO DE USO
}

\section{ANALYSIS THE CUTTING LOSS OF INSTRUMENTS PROTAPER IN RELATION THE NUMBER OF USE AND EFFECT OF STERILIZATION}

\author{
Miguel Simão Haddad Filho* \\ Sandra Marcia Habitante* \\ Luiz Carlos Laureano da Rosa*** \\ Graziela Salum**** \\ Edison Tibagy Dias Carvalho Almeida**** \\ João Marcelo Ferreira de Medeiros ${ }^{* \cdots * * *}$
}

\section{RESUMO}

\begin{abstract}
Objetivo: Este estudo propõe-se avaliar a perda de corte do instrumento rotatório ProTaper® em função do número de uso e esterilização em autoclave. Método: Foram estudados 10 canais simulados de resina acrílica transparente com ângulo de curvatura de $45^{\circ}$, valendo-se de instrumentos Protaper ${ }^{\circledR}$ de numeração S1, S2, F1, F2 e F3, substituindo o Sx pelas brocas de Largo \#1. Os blocos foram pesados em balança analítica digital, anotando-se o peso inicial. A seguir, houve o preparo com motor elétrico Endo Pro-Torque $₫$ da Driller com reverso automático submetido ao ajuste e controle da velocidade em $350 \mathrm{rpm}$, torque de $1 \mathrm{~N}$, com engate para contra-ângulo modelo $K_{a v o}{ }^{\circledR}$, tomando-se o cuidado de padronizar o tempo de ação de cada instrumento em 3 minutos. Usou-se o creme de Endo-PTC durante o preparo do canal simulado e após seu tempo de ação, lavava-se o canal com hipoclorito de sódio. Depois, o bloco era lavado com detergente neutro, seco com gaze, canal aspirado, seco com cone de papel absorvente, pesado, anotando-se o peso final. A cada uso as limas rotatórias eram limpas com gaze embebido em álcool e, posteriormente, lavadas com detergente neutro e secas também com gaze e levadas em envelope descartável para processo de esterilização em autoclave com ciclo de 20 minutos. Resultados: Os instrumentos utilizados perderam a sua capacidade de corte em função do número de uso. Conclusão: O instrumento perde a capacidade de corte em função do uso, e só a partir do sétimo uso, o instrumento ProTaper ${ }^{\circledR}$ perde consideravelmente sua efetividade, recomendando-se seu descarte. DESCRITORES: Preparo da cavidade dentária • Instrumentos odontológicos • Técnica odontológica de alta rotação.
\end{abstract}

\section{ABSTRACT}

Aims: This investigation aims to evaluate the cutting loss and sterilization of rotary ProTaper ${ }^{\circledR}$ instrument for sterilization process in autoclave. Method: Were studied ten substrate resin simulated canals blocks numbered 1 to 10 with angle of $45^{\circ}$. Before and after instrumentation were used with ProTaper ${ }^{\circledR}$ files numbering S1, S2, F1, F2, F3, is substituting for the Sx Largo drills \# 1. These blocks were weighted using an analytical digital scale model Mettler AJ 100 and following it were analyzed of the amount of resin removed in each sample were noted both initial and final weight. The instrumentation was made with Endo-Pro-Torque engine-driven Driller system with automatic reverse and speed control in $350 \mathrm{rpm}$, torque $1 \mathrm{~N}$ using stop watch for standardize the 3 minutes time of each instrument. Cream Endo-PTC was used during instrumentation e after that, washing the canals with $5 \mathrm{~mL}$ the distillate water with plastic syringe. The blocks were washed with detergent, dry with gauze, aspirated canal, dried with paper absorbent points, weighted and noted final weight. In each use the files were clean with gauze alcohol soaked and later washed with detergent and dried with gauze and put in recipient discard able for sterilization in autoclave Sercon model during 20 minutes. Results: The instruments used have lost the cutting ability in relation to the number of use. Conclusions: It's concluded that occurred a loss of efficiency of the cutting instrument, however the instrument ProTaper loses its cutting ability in use only after the seventh use and thus it's recommended its discard.

DESCRIPTORS: Dental cavity preparation - Dental instruments - Dental high-speed technique.

* Professor Assistente Mestre da Disciplina de Endodontia do Curso de Odontologia da Universidade São Francisco. Professor Responsável pelo Estágio Supervisionado da Disciplina de Clínica Integrada e Odontogeriatria da Universidade São Francisco. Professor Assistente da Disciplina de Endodontia do Curso de Odontologia da UNICASTELO. E-mail: miguel.filho@saofrancisco.edu.br

** Professora Assistente Doutora da Disciplina de Endodontia do Departamento de Odontologia da Universidade de Taubaté-UNITAU. Professora do Programa de Pós-Graduação (Mestrado e Doutorado) em Odontologia da Universidade de Taubaté. Chefe de Departamento de Odontologia da Universidade de Taubaté. E-mail: shabitante@uol.com.br

*** Professor Assistente Doutor da Disciplina de Bioestatística do Instituto Básico de Ciências Exatas da Universidade de Taubaté. Pesquisador do Núcleo de Pesquisas Econômico-Sociais da Universidade de Taubaté. Coordenador do NUPES. E-mail: laureanodarosa@gmail.com

**** Aluna do Programa de Pós-Graduação em nível de Mestrado do Departamento de Odontologia da Universidade de Taubaté - UNITAU. E-mail: grasalum@hotmail.com

***** Professor Assistente Doutor da Disciplina de Clínica Integrada do Departamento de Odontologia da Universidade de Taubaté-UNITAU. E-mail: edisontibagy@ig.com.br

****** Professor Assistente Doutor da Disciplina de Endodontia do Departamento de Odontologia da Universidade de Taubaté - UNITAU. Professor do Programa de Pós-Graduação (Mestrado e Doutorado) em Odontologia da Universidade de Taubaté. E-mail: jmedeiros@unitau.br 


\section{N T RO DUÇÃO}

A grande evolução da endodontia foi a introdução, em 1988, de limas rotatórias de níquel-titânio, já utilizadas pela indústria naval que fabricaram limas endodônticas a partir de fios ortodônticos de níquel-titânio. Esses instrumentos apresentam importantes vantagens como, por exemplo, melhor modelagem, principalmente nos canais curvos e uma diminuição significativa do tempo gasto para realizar o preparo dos canais (Walia et al. ${ }^{1}, 1988$ ).

Esses progressos permitem preparar o canal com menos dificuldade, rapidez e segurança, até mesmo diante das limitações impostas pela anatomia interna e grau de curvatura do canal radicular. Essa melhoria resultou em modificações importantes no instrumental endodôntico, a exemplo da introdução de novas matérias-primas com melhor eficiência de corte, flexibilidade e resistência à torção, que permitem modelagem adequada do canal radicular.

Esses instrumentos são fabricados por usinagem, a partir de uma haste de secção circular. Esse processo é mais preciso e permite maior variabilidade, tanto na secção transversal como no comprimento, o que possibilita maior profundidade das espiras, implicando mudanças em sua flexibilidade. Aliás, possuem duas grandes propriedades com significativa importância clínica: o efeito memória de forma e sua superelasticidade. O efeito memória de forma ocorre quando o metal é deformado a certa temperatura, de modo aparentemente permanente, e recupera sua forma original quando moderadamente aquecido, enquanto a superelasticidade é um termo utilizado para caracterizar a propriedade de certas ligas metálicas em retornarem à sua forma original, após livrar-se de uma ação (força) de deformação.

De acordo com Leonardo e Leonardo ${ }^{2}$ (2002), as ligas de níquel-titânio, quando submetidas à deformação de até $10 \%$, podem retornar à sua forma normal, sendo, portanto, recuperáveis, enquanto as limas de aço inoxidável somente retornam ao seu estado inicial quando a deformação não for superior a $1 \%$.
Quando se comparam limas \#15 fabricadas em níquel-titânio e em aço inoxidável1, observa-se que o NiTi apresenta duas ou três vezes mais flexibilidade, assim como superior é a resistência à fratura por torção cuja vantagem é gerar menos transporte do canal durante a instrumentação, segundo Serene', (1995).

Quando se confronta a efetividade do preparo automatizado, constata-se não haver diferença quanto ao número de uso do Sistema ProTaper em preparo de canais radiculares de molares, no que se refere ao acadêmico e ao especialista (Correia ${ }^{4}$, 2003).

No que se refere à segurança do sistema, autores têm avaliado o comportamento do instrumento quanto à fratura em relação ao número de vezes em que foram utilizadas as limas na instrumentação de canais simulados em blocos de resina, percebendo-se que a lima ProTaper mostrou-se segura até o quarto uso e que o maior índice de fratura ocorre nas limas de maiores conicidades no ponto de maior angulação de curvatura (Gênova et al. $\left.{ }^{5}, 2004\right)$.

Ao comparar limas FlexMaster, GT Rotary, ProFile, ProTaper e RaCe e instrumento Hedströen durante o preparo químico-cirúrgico do canal radicular, percebe-se que não ocorreu diferença estatisticamente significante entre as limas GT Rotary, FlexMaster, Hedströen e ProTaper, enquanto as limas Race deixaram remanescentes que não foram atingidos pelas outras limas. Com respeito ao tempo de preparo, a lima

FlexMaster requereu menos tempo do que todos os outros instrumentos, sendo que a lima Hedströen constituiu grupo sem significado estatístico com relação a ProTaper ou Race. Por outro lado, não ocorreu nenhuma fratura no grupo dos instrumentos Hedströen, assim como nas demais limas, porém, quatro do grupo FlexMaster fraturaram-se (Schirrmeister et al. $\left.{ }^{6}, 2006\right)$.

Recente pesquisa avaliou instrumentos rotatórios de níquel-titânio das marcas Hero, ProTaper e RaCe, utilizando como parâmetros mudanças no volume do canal, transporte e tempo de trabalho. Como recurso para medir essas características
HADDAD FILHO MS HABITANTE SM

ROSA LCL

SALUM G

ALMEIDA ETDC

MEDEIROS JMF

PERDA DE

CORTE DA LIMA

PROTAPER QUANTO

a ESTERILIZAÇÃO

e número de uso

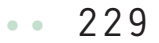

REV, ODONTOL.

UNIV, CID. São

PAULO

$2011 ; 23(3)$ : 228 -

37, SET-DEZ 
HADDAD FILHO MS HABITANTE SM ROSA LCL $S A L U M \quad G$ ALMEIDA ETDC MEDEIROS JMF

PERDA DE

CORTE DA LIMA PROTAPER QUANTO A ESTERILIZAÇÃO E NÚMERO DE USO

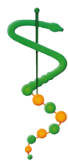

REV, ODONTOL. UNIV, CID, SÃO PAULO

2011; 23(3): 228 37, SET-DEZ
I SSN 1983-5183

foi utilizada a tomografia computadorizada em dentes extraídos. Concluiu-se que, no que diz respeito ao tempo de trabalho, não ocorreu diferença entre os sistemas e nos demais itens relacionados não houve diferença estatisticamente significante (Ozgur Uyanik et al. $\left.{ }^{7}, 2006\right)$.

Foi realizada, também, avaliação em microscopia eletrônica de varredura com três marcas de instrumentos rotatórios de níquel-titânio, EndoWave, ProTaper e Race considerando-se o efeito da limpeza em solução de hipoclorito de sódio e a esterilização por repetidas vezes em função do desgaste dos instrumentos, concluindo-se que todos apresentaram leve corrosão (Kuber et al. ${ }^{8}$, 2006).

Avaliou-se, valendo-se de microscopia eletrônica de varredura, a qualidade do acabamento superficial de instrumentos rotatórios da maneira em que estes chegam ao mercado. Foram examinados os instrumentos das marcas Profile, Protaper, Race, Hero e K3 Endo, utilizando-se amostra de 50 instrumentos escolhidos aleatoriamente, sendo estes fotomicrografados nos seus 3 milímetros finais com uma ampliação de 190 vezes. Concluiu-se que todos os instrumentos apresentaram defeitos ou falhas de fabricação no acabamento de superfície, já que nenhum instrumento apresentou defeito zero (Chianello et al. ${ }^{9}$, 2008).

Marcas de instrumentos rotatórios de níquel-titânio, Mtwo e Protaper e instrumentos manuais de níquel-titânio foram avaliados por técnicas de preparo de canais em raízes achatadas. Os resultados não mostraram diferença estatística quando comparados os sistemas rotatórios, que se mostraram superiores quando comparados às limas manuais de níquel-titânio. Concluiu-se que nenhuma técnica foi capaz de instrumentar de forma anatomicamente correta os canais radiculares; contudo, houve vantagens superiores para os sistemas rotatórios (Elayouti et al. ${ }^{10}, 2008$ ).

Utilizaram-se três marcas de instrumentos rotatórios de níquel-titânio, Protaper, R-Endo e Mtwo, para avaliação de sua capacidade quanto à eficiência na desobturação de canais em 60 dentes recém-extraídos. Os três sistemas apresentaram maior eficiência de tempo comparados ao sistema manual, porém, nenhum foi capaz de remover todo o material obturador, com vantagens para os sistemas Protaper e Mtwo (Tasdemir et al. $\left.{ }^{11}, 2008\right)$.

Embora a resina acrílica seja material de escolha em muitas investigações, outro modelo experimental pode ser utilizado como substrato, como, por exemplo, a

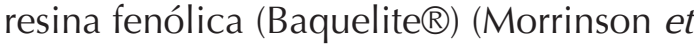
al. ${ }^{12}$ 1989; Sakane ${ }^{13}, 2007$; Alkmin et al. ${ }^{14}$ 2007; Medeiros et al. ${ }^{15}$ 2008; Alves-Claro et al. ${ }^{16}$ 2008; Reis ${ }^{17}, 2009$; Medeiros et al. ${ }^{18}$ 2009). (Morrison et al. ${ }^{12}, 1989$, Saka$\mathrm{ne}^{13}, 2007$, Alkmin et al. ${ }^{14}, 2007$, Ferreira de Medeiros et al. ${ }^{15}$, 2008, Alves-Claro et al. ${ }^{16}, 2008$, Reis ${ }^{17}, 2009$, Medeiros et al. ${ }^{18}$, 2009)

Pela primeira vez, foi realizado procedimento de uso linear contínuo mecanizado usando-se placas sulcadas de resina fenólica, para avaliação dos efeitos do emprego da esterilização na eficiência de corte de limas endodônticas de uso manual, demonstrando-se que a resina fenólica constitui um excelente substrato para o teste de corte (Morrison et al. ${ }^{12}, 1989$ ).

Aliás, autores confirmaram que o acrílico representado pelo polimetilmetacrilato, ao ser testado por meio de testes de microdureza Vickers em placas de desgaste, possui baixa dureza e alta ductibilidade respondendo ao ciclo de desgaste sem qualquer perda de peso quando sofre processo de limagem com os instrumentos endodônticos, ao contrário do que ocorre com a resina fenólica. Neste estudo, as placas de acrílico $(21,10)$ apresentaram valor médio de microdureza Vickers menor do que as placas de baquelita, cujo valor é de 39,92 (Alves-Claro et al. ${ }^{16}, 2008$ ).

Foi analisada in vitro também a capacidade de corte e deformação de limas do sistema rotatório em função de 5 usos, utilizando-se 10 limas rotatórias F2 do Sistema ProTaper Universal® e 10 limas rotatórias 25/.06 do Sistema EndoSequence $\circledR$ acionadas por motor elétrico X-SMART com torque de $2 \mathrm{~N}$, e velocidade conforme fabricante, em 20 placas sulcadas de resina fenólica com ângulo de $30^{\circ}, 3$ centímetros de comprimento por 2 centímetros de largura realizando-se cinco sulcos de 1 milímetro de profundidade nas placas. As limas ProTaper ${ }^{\circledR}$ e Endo- 
Sequence ${ }^{\circledR}$ apresentaram capacidade de corte semelhantes e, quanto à deformação de ambas as marcas, não houve diferenças entre elas (Reis ${ }^{17}, 2009$ ).

A capacidade de corte de instrumentos rotatórios $\mathrm{Ni}$-Ti depende da configuração experimental do substrato, do desenho do instrumento e da condição de corte (Shen e Haapasalo ${ }^{19}$, 2008).

Por outro lado, devido ao alto custo, esses instrumentos geralmente são reutilizados, o que torna essencial seguir criteriosamente os procedimentos de controle de infecção (O'Hoy et al. $\left.{ }^{20}, 2003\right)$.

Claro está que esses instrumentos são submetidos a vários ciclos de esterilização após subsequentes usos, possibilitando o aumento da taxa de sucesso dos tratamentos endodônticos.

Nesse particular, relatos existem da reutilização das limas na preparação de canais radiculares, o que torna necessárias repetidas exposições à esterilização entre os usos. Com isso, a flexibilidade, resistência à fratura por tração e eficiência de corte, não deveriam ser significantemente alteradas pelos procedimentos de esterilização o que asseguraria seu uso mesmo nas limas previamente usadas, além de permanecerem efetivas na limpeza e preparação de canais (Canalda-Sahli et al. ${ }^{21}$, 1998).

Apesar disso, para remoção completa de matéria orgânica e debris acumulados, devem ser realizados procedimentos efetivos de limpeza previamente aos processos de esterilização.

Assim é que as condições laboratoriais e a sequência de procedimentos de limpeza incluem armazenamento dos instrumentos em meio úmido, enquanto aguardam a limpeza, escovação, imersão em hipoclorito de sódio 1\% e limpeza em cuba ultrassônica, para a total remoção dos debris orgânicos. Entretanto, durante a prática clinica, esse protocolo reduziu substancialmente a contaminação biológica, mas a limpeza completa foi alcançada em apenas $87 \%$ dos casos (Linsuwanont et al. ${ }^{22}, 2004$, Parashos et al. $\left.{ }^{23}, 2004\right)$.

Não obstante o hipoclorito de sódio ser eficiente na remoção de matéria orgânica, pois, apresenta propriedades viruscida e bactericida, por outro lado, é altamente corrosivo aos metais, podendo causar a deterioração dos instrumentos endodônticos e potencialmente enfraquecer a estrutura dos mesmos (O'Hoy et al. ${ }^{20}, 2003$ ).

Os métodos de esterilização que envolvem os processos físicos, particularmente o calor úmido em autoclave e o calor seco em estufa, representam os mais frequentes métodos de esterilização utilizados nas clínicas odontológicas (Samaranayake et al. ${ }^{24}, 1995$, Estrela e Estrela $\left.{ }^{25}, 2005\right)$.

Foi feita uma avaliação sobre a reutilização de instrumentos rotatórios de níquel-titânio das marcas Alpha File, FlexMaster, K3, Mtwo, ProTaper, ProFile e Race após uso em canais de dentes extraídos e, em seguida, submetidos à imersão por 24 horas em solução de hidróxido de cálcio e hipoclorito de sódio, lavados em cubas ultrassônicas e esterilizados. Concluiu-se que todas as soluções não foram capazes de limpar completamente os restos orgânicos dos instrumentos, além de se observar significativa corrosão nos instrumentos do grupo submetido ao hipoclorito de sódio e restos de matéria orgânica na maioria deles (Sonntag e Peters ${ }^{26}$, 2007).

Por outro lado, o ciclo de esterilização, quer seja em autoclave ou em estufa, consiste de um período de aquecimento, tempo de manutenção e tempo de resfriamento. (Samaranayake et al. ${ }^{24}$, 1995). A autoclave (calor úmido sob pressão) é o método mais rápido, visto que, em virtude da pressão, apresenta um maior poder de penetração. Além disso, a umidade catalisa a coagulação das proteínas, que constitui o mecanismo de ação desse método. A autoclave convencional exige um tempo de 20 minutos a $121^{\circ}$ sob pressão de 15 libras, porém, o ciclo para esterilização necessita de um tempo total de, no mínimo, 90 minutos (Estrela e Estrela $\left.{ }^{25}, 2005\right)$.

Da mesma forma, acentuam Reams et al. ${ }^{27}$ (1995) que períodos mais longos podem ser necessários para que o vapor alcance efetivamente o centro de pacotes muito grandes; entretanto, um período de 30 minutos normalmente é adequado. Entretanto um dos inconvenientes provocados pela autoclave convencional é a corrosão nos instrumentos metálicos, devido ao alto teor de oxigênio presente nesses aparelhos (Estrela e Estrela ${ }^{25}$, 2005).
HADDAD FILHO MS

HABITANTE SM

ROSA LCL

SALUM G

ALMEIDA ETDC

MEDEIROS JMF

PERDA DE

CORTE DA LIMA

PROTAPER QUANTO

a ESTERILIZAÇÃO

e NúMERo de USO

$\therefore 231$

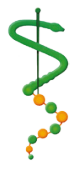

REV, ODONTOL.

UNIV, CID, SÃO

PAULO

$2011 ; 23(3)$ : 228 -

37, SET-DEZ 
HADDAD FILHO MS

HABITANTE SM

ROSA LCL

$S A L U M \quad G$

ALMEIDA ETDC

MEDEIROS JMF

PERDA DE

CORTE DA LIMA

PROTAPER QUANTO

A ESTERILIZAÇÃO

E NÚMERO DE USO

$232 \ldots$

REV. ODONTOL.

UNIV. CID, SÃO

PAULO

$2011 ; 23(3): 228-$

37, SET-DEZ
Sistemas como ProTaper (Dentsply Maillefer, Ballaigues, Suíça), ProTaper de uso manual (Dentsply Maillefer) e K3 (SybronEndo, Orange, CA) em recente análise foram descartados em 3 clínicas endodônticas. Os instrumentos foram avaliados em função dos defeitos e fatores que levam ao instrumento a deformação ou fratura. Um total de 1682 instrumentos foi recolhido e examinado entre janeiro de 2006 a abril de 2007 e registrou-se a ocorrência de defeito. A prevalência de defeitos como alongamento ou estiramento das espiras da lima e fratura foi de 3\% e 5\%. A ProTaper apresentou o defeito de taxa de fratura e distorção em $7 \%$ na clínica A e 13\% na clínica B. A menor taxa de defeito tipo estiramento foi detectada nas limas K3, $1 \%$, e, para fratura, $3 \%$. Os defeitos das limas de níquel-titânio foram influenciados por fatores tais como operador, a técnica de preparo e o desenho do instrumento (Shen et al.28, 2009).

Importa notar que as superfícies de corte das lâminas de limas de níquel-titânio se deformam durante usos subsequentes, o que reduz o seu poder de corte, e para mais, ao submeterem-se aos processos de limpeza e esterilização também sofrem corrosão. O propósito desta presente investigação foi avaliar a perda de corte do instrumento rotatório ProTaper $\AA$ em função do número de uso valendo-se de processo de esterilização em autoclave.

\section{MATERIAL E MÉTODOS}

Foram utilizados 10 canais simulados em resina acrílica transparente com ângulo de curvatura do conduto de $45^{\circ}$ e instrumentos Protaper ${ }^{\circledR}$ de numeração S1, S2, F1, F2 e F3, considerando-se a substituição do $S x$, também constituinte dessa série, pelas brocas de Largo \#1, em função do alto índice de fratura.

As amostras foram numeradas de $1 \mathrm{a}$ 10, pesadas cada uma em balança analítica de máxima precisão modelo Mettler AJ 100, considerando-se como peso inicial (P0) anotado em ficha. Essas amostras foram acondicionadas em embalagens plásticas de $10 \times 5$.

A seguir, cada bloco foi preso em uma morsa para execução do preparo, evitando-se, sempre que possível, o contato ma- nual.

O preparo foi realizado às custas de motor elétrico Endo Pro-Torque ${ }^{\circledR}$ da Driller com reverso automático, submetido ao ajuste e controle da velocidade em 350 rpm, torque de $1 \mathrm{~N}$, com engate para contra-ângulo modelo Kavo®.

Com auxílio de cronômetro, tomou-se o cuidado de padronizar o tempo de ação de cada instrumento endodôntico em 3 minutos, valendo-se a cada mudança de instrumento de creme de Endo PTC como lubrificante durante o preparo do canal simulado e, decorrido este tempo, lavava-se o canal com 5 mililitros de hipoclorito de sódio a $1 \%$ com seringa plástica.

Concluída a sequência operatória, o bloco era lavado com detergente neutro e seco com gaze, realizando-se, no canal, aspiração e secagem com cone de papel absorvente taper.06 e novamente pesado, constituindo o peso final (P1) que era anotado na ficha.

Tal protocolo foi repetido até a intervenção do último bloco do canal simulado, ou seja, limpeza e pesagem inicial do bloco de canal simulado número 1 , fixação do bloco em morsa, preparo da entrada do canal com brocas de Largo \#1 durante 3 minutos, ação do instrumento S1 acionado a motor elétrico, com velocidade de 350 rpm e torque de $1 \mathrm{~N}$, por 3 minutos, lavagem do canal com $5 \mathrm{ml}$ de água destilada e aplicação de lubrificante, ação do instrumento S2 acionado a motor elétrico, com velocidade de $350 \mathrm{rpm}$ e torque de $1 \mathrm{~N}$, por 3 minutos, lavagem do canal com $5 \mathrm{ml}$ de água destilada e aplicação de lubrificante, ação do instrumento F1 acionado a motor elétrico, com velocidade de 350 rpm e torque de $1 \mathrm{~N}$, por 3 minutos, lavagem do canal com $5 \mathrm{ml}$ de água destilada e aplicação de lubrificante, ação do instrumento F2 acionado a motor elétrico, com velocidade de 350rpm e torque de $1 \mathrm{~N}$, por 3 minutos, lavagem do canal com $5 \mathrm{ml}$ de água destilada e aplicação de lubrificante, ação do instrumento F3 acionado a motor elétrico, com velocidade de 350 rpm e torque de $1 \mathrm{~N}$, por 3 minutos.

Em continuidade, lavagem do conduto com hipoclorito de sódio a $1 \%$ e aspiração, remoção do bloco de acrílico da morsa, lavagem do bloco com detergente 
neutro, secagem do bloco com gaze, secagem do conduto com cone de papel absorvente, pesagem final do bloco na balança de precisão.

Após isso, computava-se a perda de peso pela subtração do peso inicial menos final, com auxílio de calculadora.

Cumpre assegurar que, antes de cada utilização, as limas eram inicialmente limpas com gaze embebido em álcool e, posteriormente, lavadas com detergente neutro e secas também com gaze, a fim de acomodá-las em envelope descartável para serem submetidas ao processo de esterilização em autoclave com ciclo de 20 minutos.

A seguir, os dados foram tabulados por meio do programa BioEstat versão 5.0 e, servindo-se do gráfico de linha, foi analisada a perda de corte em função do número de uso. Média, desvio-padrão e coeficiente de variação foram utilizados, bem como o teste paramétrico " $\mathrm{t}$ " Student, ao nível de $5 \%$, para comparar a perda média de corte entre os 5 primeiros e os 5 últimos usos.

\section{RESULTADOS}

Os resultados desta presente investigação encontram-se expressos nas Tabelas 1 e 2 e no Gráfico 1.

A perda média de corte do instrumento rotatório ProTaper® nos 5 últimos usos são significativamente menores do que nos 5 primeiros usos $(t=6,3357$ - Pvalor
= 0,0002), o que traduz que o instrumento está perdendo poder de corte conforme o aumento no número de uso.

\section{I SCUSSÃO}

O preparo do canal radicular constitui uma das etapas mais importantes da terapia endodôntica e para tal é necessário o uso de técnica adequada, instrumento flexível de reconhecida capacidade de corte, devendo-se reunir, ao mesmo tempo, uso de substância química auxiliar e habilidade profissional.

De outra maneira, para escolha de técnica adequada à instrumentação de canais curvos, deve-se levar em conta o alargamento cervical prévio ao preparo do canal. Tais procedimentos propiciam criação de uma zona de escape na região cervical, possibilitando, durante a irrigação-aspiração, maior arraste e refluxo de magma e raspas de dentina que foram cor-

Gráfico 1 - Perca de corte do instrumento

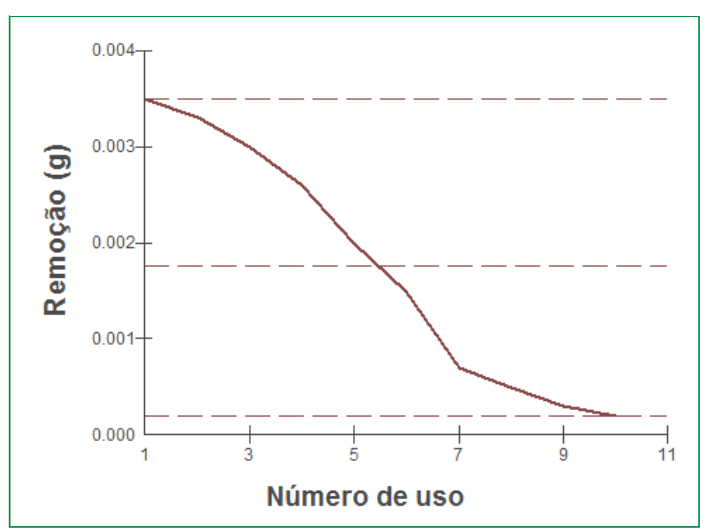

HADDAD FILHO MS HABITANTE SM

ROSA LCL

$S A L U M \quad G$

ALMEIDA ETDC

MEDEIROS JMF

PERDA DE

CORTE DA LIMA

PROTAPER QUANTO

A ESTERILIZAÇÃO

E NÚMERO DE USO

Tabela 1 - Análise da remoção de material de cada bloco em gramas bem como a diferença entre peso inicial e peso final de cada amostra em miligramas, em função do número de uso.

\begin{tabular}{cccc}
\hline \hline AMOSTRA & PESO INICIAL (P0) & PESO FINAL (P1) & DIFERENÇA DE PESO (P0-P1) \\
\hline 01 & 3,3788 & 3,3753 & 3,5 \\
02 & 3,4036 & 3,4003 & 3,3 \\
03 & 3,3832 & 3,3802 & 3,0 \\
04 & 3,3950 & 3,3924 & 2,6 \\
05 & 3,3775 & 3,3755 & 2,0 \\
06 & 3,3834 & 3,3819 & 1,5 \\
07 & 3,3950 & 3,3943 & 0,7 \\
08 & 3,3927 & 3,3922 & 0,5 \\
09 & 3,4049 & 3,4046 & 0,3 \\
10 & 3,3981 & 3,3979 & 0,2 \\
\hline \hline
\end{tabular}

$\mathrm{t}=6,3357($ Pvalor $=0,0002)$ 
HADDAD FILHO MS HABITANTE SM ROSA LCL $S A L U M \quad G$ ALMEIDA ETDC MEDEIROS JMF PERDA DE CORTE DA LIMA PROTAPER QUANTO A ESTERILIZAÇÃO E NÚMERO DE USO

\section{$234 \cdots$}

REV, ODONTOL.

UNIV. CID. SÃO

PAULO

$2011 ; 23(3): 228-$

37, SET-DEZ

Tabela 2 - Média (), Desvio-padrão () e Coeficiente de variação (CV) dos 5 primeiros usos em relação aos 5 últimos usos em termos de remoção (mg).

\begin{tabular}{llll}
\hline \hline GRUPO & $\bar{X}$ & $\sigma$ & CV $(\%)$ \\
\hline 1 a 5 & 2,88 & 0,60 & 20,83 \\
6 a 10 & 0,64 & 0,52 & 81,25 \\
\hline \hline
\end{tabular}

tadas. Assim, recomenda-se alargamento progressivo da entrada do canal radicular antes da instrumentação para remover essas interferências, facilitando a manobra.

Com vistas a isso, a intenção deste trabalho foi avaliar a perda de corte de um tipo de instrumento de níquel-titânio ProTaper do sistema rotatório em função do número de uso, valendo-se, como processo de esterilização, da autoclave.

Inicialmente, realizou-se preparo químico-cirúrgico valendo-se da lima ProTaper em todas as amostras dos canais simulados, começando no bloco 1 e terminando no bloco 10 (Tabela 1).

A esse respeito, o exame da tabela revela os pesos iniciais e finais dos blocos, em gramas, antes e depois da instrumentação rotatória bem como a diferença entre os pesos inicial (P0) e final (P1) com a lima ProTaper. Esclareça-se que os pesos iniciais mencionados nesta tabela foram registrados depois do preparo da entrada do canal simulado (preparo cervical) com brocas de Largo \#1, durante 3 minutos. Além do mais, com respeito à Tabela 1 , verificou-se diferença dos pesos iniciais e finais em gramas em todas as amostras.

Mais ainda, os resultados das diferenças de pesos iniciais e finais, ou seja, a remoção do material desta atual pesquisa foi menor em termos quantitativos quando comparada com a remoção de material dos blocos fornecidos por outros autores (Ferreira de Medeiros et al. ${ }^{15}$, 2008, Mansano $^{29}, 2009$ ).

Assim, ao visualizarmos as tabelas dos autores anteriormente citados, verifica-se que, apesar de terem utilizado tempos diferentes (6 e 18 minutos) daqueles usados nesta pesquisa (15 minutos), a quantidade de material removido foi menor, sendo que no trabalho de Medeiros et al. ${ }^{15}$ (2008) não foi realizado preparo cervical, ao contrário de Mansano ${ }^{29}$ (2009) que assim o fez.
Outra diferença a se considerar foi o tipo de instrumento utilizado durante o preparo do canal, sendo a lima Flexofile de aço inoxidável usada manualmente e acoplada ao sistema rotatório Endo-Gripper (Medeiros et al. ${ }^{15}$ (2008), enquanto Mansano $^{29}$ (2009) valeu-se de lima tipo K associada ou não à lima tipo Hedströen, ambas de níquel-titânio e ambas acopladas ao sistema rotatório Endo-Gripper.

Um ponto em comum em ambos os trabalhos acima comentados foi o tipo de amostra utilizada nos ensaios, ou seja, blocos de canais simulados à base de resina fenólica, ao contrário da presente pesquisa, que usou blocos de canais simulados à base de resina acrílica.

O fato de a resina acrílica apresentar baixa dureza e se deformar com a ação dos instrumentos endodônticos, ao invés de se desgastar, talvez signifique que esse material, ao sofrer processo de limagem, ao contrário do que ocorre com a resina fenólica, não perde peso (Alves-Claro et al. $\left.{ }^{16}, 2008\right)$. Neste estudo, as placas de acrílico apresentaram valor médio de microdureza Vickers $(21,10)$ menor do que as placas de resina fenólica $(39,92)$, praticamente o dobro de dureza.

Não obstante, a capacidade de corte de instrumentos rotatórios $\mathrm{Ni}$-Ti depende da configuração experimental do substrato, do desenho do instrumento e da condição de corte (Shen e Haapasalo ${ }^{19}$, 2008). Isso não quer dizer que a resina acrílica não represente substrato para ensaios com instrumentos endodônticos; todavia, o ideal seria a escolha de resina fenólica, que é em dobro mais dura (dureza Vickers) do que a resina acrílica e com valor mais próximo à dentina humana $\left(57-60 \mathrm{Kgf} / \mathrm{mm}^{2}\right)$ (Anusavice e Antonson ${ }^{30}$, 2005).

Diga-se a propósito que, durante o preparo de canais simulados em blocos de resina fenólica, há ocorrência de desgaste do material que está sendo cortado e sub- 
sequente perda de peso em resposta ao ciclo de limagem e não deformação plástica (resina acrílica) e, por essa razão, nas pesquisas onde foram realizados ensaios em canais simulados com resina fenólica (Ferreira de Medeiros et al. ${ }^{15}, 2008$, Reis 17, 2009, Mansano ${ }^{29}$, 2009), a remoção foi maior comparativamente com os achados providenciados nesta presente investigação; apresentar perda de peso em resposta ao ciclo de limagem, o que constitui requisitos indispensáveis na escolha desta substância para preparação do canal radicular.

Convém observar que na Tabela 1 constata-se diferença dos pesos iniciais e finais em gramas das amostras testadas. Tanto a pesagem antes do preparo do canal como a pesagem posterior ao preparo expressam, de modo convincente, o efeito da remoção quantitativa da instrumentação, e tal ocorrência mostra ainda que os instrumentos na medida em que eram utilizados iam perdendo gradativamente o seu poder cortante, isto é, valor máximo de corte para o bloco 1 (3,5mg) e valor mínimo para o bloco $10(0,2 \mathrm{mg})$, fato este notado também no Gráfico 1 , o que demonstra ocorrência de remoção do material cortado das paredes dos canais simulados durante a instrumentação.

Realmente, ao se observar a Tabela 1 , verifica-se que os instrumentos de primeiro uso removeram maior quantidade de material em gramas, do que os instrumentos de usos subsequentes: a exemplo das amostras 1 e 10. Nota-se decréscimo progressivo no que respeita à remoção do material do canal simulado em gramas, acontecimento este verificado nas últimas amostras de cada grupo, onde as diferença foram bem menores. Esse substrato com canais simulados à base de resina acrílica utilizado é um protótipo cuja geometria e dureza são semelhantes entre si e com mesmo grau de curvatura, o que possibilitou a ação do instrumento endodôntico mais uniforme nas amostras utilizadas.

Assim, os graus de curvaturas fornecidos neste trabalho foram os mesmos para todos os blocos de canais simulados, isto é, $45^{\circ}$, e diferem de outras metodologias que utilizam dentes humanos com indicadores de dureza diferentes nos três terços do canal radicular e graus de curvaturas variáveis.

Na verdade, é importante recomendar, sempre que possível, instrumento adequado e de primeiro uso, devido à ocorrência de perda de corte, especialmente nos que foram utilizados sete vezes e perderam, de acordo com os dados da Tabela 1, cerca de $80 \%$ da capacidade de corte, enquanto uma redução de cerca de $5 \%$ de perda da capacidade de corte ocorre nos instrumentos usados uma vez.

Entende-se, dessa maneira, que o uso continuado dos instrumentos deve, segundo este protocolo, ser descartado, notadamente já a partir do sétimo uso, pois, além da reduzida capacidade de corte, há enfraquecimento de lâminas cortantes e subsequente fratura e, além do mais, presume-se que a perda de corte em função de múltiplos usos acontece também em decorrência do desgaste das lâminas durante os processos de esterilizações, sobretudo, o calor úmido proporcionado pela autoclave que é um método mais rápido apresentando maior poder de penetração graças à pressão por um período de 30 minutos.

Um dos inconvenientes provocados pela autoclave convencional é a corrosão nos instrumentos metálicos, devido ao alto teor de oxigênio presente nesses aparelhos (Estrela e Estrela $\left.{ }^{25}, 2005\right)$, sem contar com a corrosão que o hipoclorito de sódio provoca sobre a superfície da liga metálica dos instrumentos (O'Hoy et al. ${ }^{20}$, 2003), deixando-os mais frágeis.

\section{CONCLUSÕES}

De posse dos resultados obtidos, no que diz respeito à perda de corte, parece adequado concluir que, a cada uso, o instrumento perde sutilmente sua capacidade de corte; entretanto, só a partir do sétimo uso precedido pelo processo de esterilização por calor úmido, o instrumento rotatório Protaper ${ }^{\circledR}$ perde consideravelmente sua efetividade e, nesse instante, recomenda-se seu descarte.
HADDAD FILHO MS

HABITANTE SM

ROSA LCL

SALUM G

ALMEIDA ETDC

MEDEIROS JMF

PERDA DE

CORTE DA LIMA

PROTAPER QUANTO

a ESTERILIZAÇÃO

e número de uso

$\therefore 235$

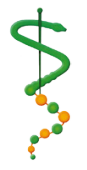

REV, ODONTOL.

UnIV. CID. São

PAULO

2011; 23(3): 228 -

37, SET-DEZ 
HADDAD FILHO MS HABITANTE SM ROSA LCL $S A L U M \quad G$ ALMEIDA ETDC MEDEIROS JMF PERDA DE CORTE DA LIMA PROTAPER QUANTO A ESTERILIZAÇÃO E NÚMERO DE USO

REV. ODONTOL. UNIV. CID. SÃO PAULO $2011 ; 23(3): 228-$ 37, SET-DEZ

\section{REFERÊNCIAS}

1. Walia HM, Brantley WA, Gerstein H. An initial investigation of the bending and torsional properties of Nitinol root canal files. J Endod 1988 Jul;14(7):346-51.

2. Leonardo M, Leonardo M. Aprimoramento e simplificação de técnicas endodônticas. In: Leonardo M, Leonardo M. Sistema rotatório em endodontia, instrumentos de níquel-titânio. São Paulo: Artes Médicas; 2002. p. 23-7.

3. Serene T. Nickel-titanium instruments: aplications in endodontics. St. Louis: Ishiyaku EuroAmerica; 1995.

4. Correia D. Avaliação do numero de uso do sistema Protaper®, no preparo químico-cirurgico de molares, quando executado por um acadêmico e por um especialista [Mestrado]. São Paulo: Faculdade de Odontologia da Universidade de São Paulo; 2003.

5. Gênova APS, Antonio MPS, Davidowicz H, Moura AAM. Avaliação do índice de fraturas das limas ProTaper em canais simulados Rev Inst Ciênc Saúde 2004 jan.-mar;22(1):51-4.

6. Schirrmeister JF, Strohl C, Altenburger MJ, Wrbas KT, Hellwig E. Shaping ability and safety of five different rotary nickel-titanium instruments compared with stainless steel hand instrumentation in simulated curved root canals. Oral Surg Oral Med Oral Pathol Oral Radiol Endod 2006 Jun;101(6):807-13.

7. Ozgur Uyanik M, Cehreli ZC, Ozgen Mocan B, Tasman Dagli F. Comparative evaluation of three nickel-titanium instrumentation systems in human teeth using computed tomography. J Endod 2006 Jul;32(7):668-71.

8. Kuber S, Mohan B, Lakshminarayanan L. Effect of cleaning and sterilization procedures on niti rotary files: an SEM and EDS study. Endodontol 2006 18(1):34-41.

9. Chianello G, Specian VL, Hardt LC, Raldi DP, Lage-Marques JL, Habitante SM. Surface finishing of unused rotary endodontic instruments: a SEM study. Braz Dent J 2008 19(2):109-13.

10. Elayouti A, Chu AL, Kimionis I, Klein C, Weiger R, Lost C. Efficacy of rotary instruments with greater taper in preparing oval root canals. Int Endod J 2008 Dec;41(12):108892.

11. Tasdemir T, Er K, Yildirim T, Celik D. Efficacy of three rotary NiTi instruments in removing gutta-percha from root canals. Int Endod J 2008 Mar;41(3):191-6.

12. Morrison SW, Newton CW, Brown CE, Jr. The effects of steam sterilization and usage on cutting efficiency of endodontic instruments. J Endod 1989 Sep;15(9):427-31.

13. Sakane F. Avaliação in vitro do desgaste e da capacidade de corte de limas manuais de NI-TI [Mestrado]. Taubaté: Departamento de Odontologia da Universidade de Taubaté; 2007.

14. Alkmin S, Pinto C, Habitante S, Zöllner N, Medeiros J, Lage-Marques J. Concepção de um modelo experimental a base de resina fenólica utilizado em endodontia. Braz Oral Res 2007 21(Suppl.1):284-344.

15. Ferreira de Medeiros JM, Zöllner NA, de Carvalho PL, Rosifini Alves AP, Pires Clemente RG. Capacidad de corte de la lima Flexofile en canales simulados. Rev Cubana Estomatol 200845.

16. Alves-Claro AP, Claro FA, Uzumaki ET. Wear resistance of nickel-titanium endodontic files after surface treatment. J Mater Sci Mater Med 2008 Oct;19(10):3273-7. 
17. Reis P. Avaliação in vitro da capacidade de corte e deformação de limas rotatórias de níquel-titânio [Mestrado]. Taubaté: Departamento de Odontologia da Universidade de Taubaté; 2009.

18. Medeiros JMF, Rodrigues GdA, Santos ACdM, Rosa LCL, Carvalho PL, Nohara EL. Determinação do comprimento e grau de curvatura em canais radiculares curvos simulados confeccionados em blocos de resina fenólica. Rev odontol Univ Cid Sao Paulo 2009 set.-dez;21(3):

19. Shen Y, Haapasalo M. Three-dimensional analysis of cutting behavior of nickel-titanium rotary instruments by microcomputed tomography. J Endod 2008 May;34(5):606-10.

20. O'Hoy PY, Messer HH, Palamara JE. The effect of cleaning procedures on fracture properties and corrosion of NiTi files. Int Endod J 2003 Nov;36(11):724-32.

21. Canalda-Sahli C, Brau-Aguade E, Sentis-Vilalta J. The effect of sterilization on bending and torsional properties of K-files manufactured with different metallic alloys. Int Endod J 1998 Jan;31(1):48-52.

22. Linsuwanont $\mathrm{P}$, Parashos $\mathrm{P}$, Messer $\mathrm{HH}$. Cleaning of rotary nickel-titanium endodontic instruments. Int Endod J 2004 Jan;37(1):19-28.

23. Parashos $\mathrm{P}$, Linsuwanont $\mathrm{P}$, Messer $\mathrm{HH}$. A cleaning protocol for rotary nickel-titanium endodontic instruments. Aust Dent J 2004 Mar;49(1):20-7.

24. Samaranayake L, Scheutz F, Cottone J. Controle da infecção para a equipe odontológica. 2.ed. ed. São Paulo: Santos; 1995.

25. Estrela C, Estrela C. Control de Infección en odontología. São Paulo: Artes Médicas; 2005.

26. Sonntag D, Peters OA. Effect of prion decontamination protocols on nickel-titanium rotary surfaces. J Endod 2007 Apr;33(4):442-6.

27. Reams GJ, Baumgartner JC, Kulild JC. Practical application of infection control in endodontics. J Endod 1995 May;21(5):281-4.

28. Shen Y, Haapasalo M, Cheung GS, Peng B. Defects in nickel-titanium instruments after clinical use. Part 1: Relationship between observed imperfections and factors leading to such defects in a cohort study. J Endod 2009 Jan;35(1):129-32.

29. Mansano T. Estudo comparativo "in vitro" da capacidade de corte de dois tipos de limas de níquel-titânio após o preparo de canais radiculares simulados [Trabalho de conclusão de curso]. Taubaté: Departamento de Odontologia da Universidade de Taubaté; 2009.

30. Anusavice K, Antonson S. Materiais de acabamento e polimento. In: Anusavice K. Phillips Materiais dentários. 11.ed. ed. Rio de Janeiro: Elsevier; 2005.

Recebido em 05/09/2011

Aceito em 15/09/2011

HADDAD FILHO MS

HABITANTE SM

ROSA LCL

SALUM G

ALMEIDA ETDC

MEDEIROS JMF

PERDA DE

CORTE DA LIMA

PROTAPER QUANTO

A ESTERILIZAÇÃO

E NÚMERO DE USO

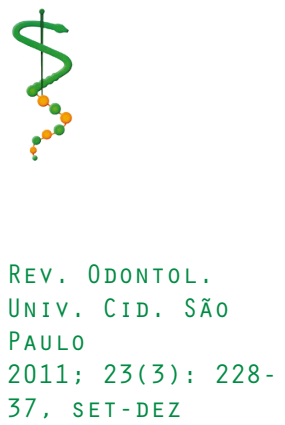

